\title{
Non Informative Priors for the Stress-Strength Reliability in the Generalized Augmented Inverse Gaussian Distribution
}

\author{
N. CHANDRA ${ }^{1}$ and V. K. RATHAUR \\ Department of Statistics, Ramanujan School of Mathematical Sciences, \\ Pondicherry University, Puducherry-605 014, India
}

(Received on September 30, 2016, revised on November 25, 2016)

\begin{abstract}
In this paper the Bayesian and classical estimation of augmented strength reliability under Augmentation Strategy Plan (ASP) have been considered. The Augmentation Strategy Plan (ASP) is suggested for enhancing the strength of failed equipment. The Bayes estimation is carried out by assuming the model parameters are random variable and having non-informative type of priors (uniform and Jeffery's priors) under two different loss functions, viz. squared error loss function (SELF) and Linex loss function (LLF). We assume that the Inverse Gaussian stress $(\mathrm{Y})$ is subjected to equipment having Inverse Gaussian strength $(\mathrm{X})$ and are independent to each other. A comparative study between ML and Bayesian estimators have been carried out on the basis of mean square errors (MSE) and absolute biases. The Markov Chain Monte Carlo method of approximations has been applied to draw posterior expectations under both the loss functions. The MSE and absolute biases are calculated with 1000 replications.
\end{abstract}

Keywords: Augmenting Strength reliability, Inverse Gaussian distribution, Maximum likelihood estimation, Bayes estimation, Metropolis-Hasting algorithm.

\section{Introduction:}

The two-parameter Inverse Gaussian (IG) distribution is a positively skewed distribution which arises as the first passage time distribution in a Brownian motion process with positive drift. The IG distribution is being used for reliability and life testing problems where the initial failure rate is high and it is proposed as an alternative to the Weibull, Lognormal and other similar life distributions. Many researchers like [21, 2, 14, 22 and 26] have been focused on IG distribution and its characterization in context of failure time analysis for reliability prediction. One may also refer to [27, 28] and [11, 12 and 13] for various applications of IG distribution including its sampling inferences. Some other areas are also identified where the real life applications of IG distribution are found e.g., theoretical physics to meteorology, sequential analysis, industrial quality control and business applications etc. (see; [19]). The probability density function (pdf) of IG distribution is given by

$$
f_{X}(x)=\sqrt{\frac{\lambda}{2 \pi x^{3}}} \exp -\left\{\frac{\lambda(x-\mu)^{2}}{2 \mu^{2} x}\right\} ; \quad x \geq 0, \mu>0, \lambda>0
$$

and it is denoted by $I G(\mu, \lambda)$ with mean $\mu$ and variance $\mu^{3} / \lambda$.

\footnotetext{
${ }^{1}$ Communicating author's email: nc.stat@gmail.com
} 
The stress-strength reliability generally assesses the reliability of an equipment/system in terms of random variables. The stress-strength reliability is defined as the probability that the system will survives its usual life by performing its intended function, provided that the random strength $(X)$ should be higher than the random stress (Y), i.e., $R=P(X>Y)$. A very recent published literature work on stress-strength system reliability problems may be referred to $[24,25]$ and references therein. As far as IG distribution is concern in context of stress-strength setup, a very few attempts have been made by the researchers. The paper [3] introduced a dynamic approach to stressstrength model with the time varying system reliability in the presence of stress process $X(t)$, which is faced by the system at time $t$ with its strength process $Y(t)$. In the similar fashion authors $[4,15]$ have also attempted the problem of modeling the stressstrength system reliability in the presence of Brownian process. To the best of our literature review there do not exist any work on parameter estimation of stress-strength reliability of IG distribution under static system approach due to its complicated expressions of distribution function. In this paper we therefore consider the classical static approach of modelling stress-strength systems for IG distribution. Here we use static in the sense that the potential data on stress $(\mathrm{Y})$ and strength $(\mathrm{X})$ are collected without depending on time. We assume that the stress (Y) and strength (X) are independent and identically distributed random variables which follow two-parameter Inverse Gaussian distribution (IGD) with probability density function (PDF) is given as in equation (1). The intension behind choosing two-parameter IG distribution is its reproductive property which leads the application of Augmentation Strategy Plan (ASP) to enhance (or augment) the system strength reliability.

In fact, ASP deals with two most important issues which are being faced by manufacturing industry/company over the last several decades. The first one is that the unusual failures problem in newly manufactured system/item/equipment while first or subsequent use and second one is that the problem of frequent failures occurs in used system. Such types of failures are known as irrelevant (or unusual) failure. An experimenter cannot perform life testing experiment for collecting the life time data under such circumstances until unless equipments are free from such types of problems. In general, any system/equipment should perform its intended function for the specific period of time. ASP may capable to protect from irrelevant failures and restore to survive at least its usual life by enhancing strength reliability of such weaker equipments.

The proposed ASP comprises three possible cases to enhance the strength reliability of an equipment to face the common stress. It is noticed that case-I and case-II of ASP were special cases of case-III, which we call it as generalized case of ASP. The different cases and its detailed discussion of ASP by assuming stress and strength are independently and identically distributed as IG distribution may be referred [8]. The problem of augmenting strength reliability was firstly attempted by [1]. They initially derived augmenting strength reliability models for exponential distribution for three different possible cases of ASP. After one decade, [7] pointed out applicability of ASP and attempted augmenting gamma strength reliability of equipment for same set up. Some more references in this direction may be viewed in $[9,10]$.

A numerous works on classical and Bayesian inferences on the parameters of IG distribution and its reliability function have taken place during the last five decades. A complete literature review in this context including a detail discussions about facts and findings are extensively reported by [23] and references therein. They attempted the ML 
and Bayesian point and interval estimation of parameters of IG distribution via numerical approximation techniques viz., Lindley approximation and Gibbs sampler methods. In this article, we consider estimation of parameters of augmented IG strength reliability models under ASP through Bayesian and ML methods. Bayesian estimates are derived under squared error loss function (SELF) and linex loss function (LLF) by assuming the uniform and Jaffrey's priors under non-informative prior set up for both the parameters $\mu$ and $\lambda$. The numerical comparison among the estimators of augmented strength reliability is carried out on the basis of MSE and absolute biases.

The rest of article is organized as follows. In section 2, a generalized form of augmented strength reliability models under ASP are introduced. A generalized form of ML estimators of augmented strength reliability under ASP is presented in section 3. In section 4, we propose a generalized form of Bayes estimation of augmented strength reliability parameters for both the prior (uniform and Jeffrey's) under both the loss functions. In section 5, the proposed methodologies are validated through simulated and real life data analysis. A simulation study and its discussions are reported based on findings of case-III under ASP in section 6. Finally, the conclusions are given in section 7.

\section{Notations:}

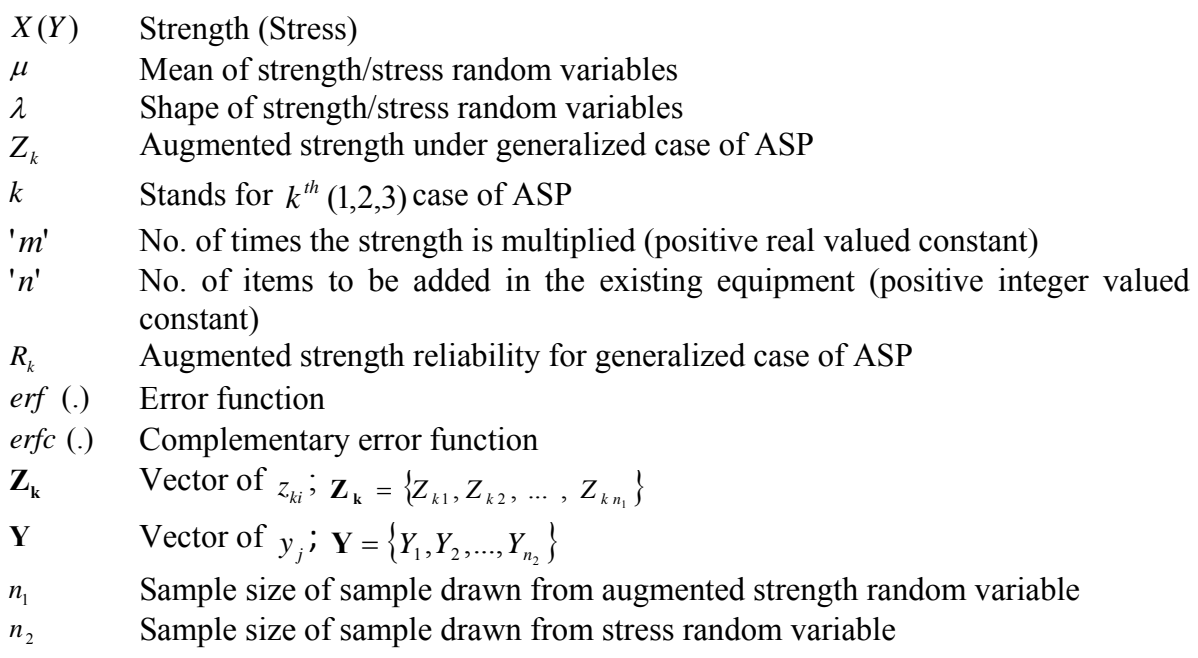

\section{A Generalized form of Augmented Strength Reliability Models:}

In this section, we tried to compute a general form of augmented strength reliability model under the generalized case (i.e. case-III) of ASP by assuming that both strength (X) and stress (Y) are independently and identically distributed as IG distribution with parameters $\mu$ and $\lambda$. Thus, under the generalized case ASP, the enhanced strength of the equipment $Z_{k}=\sum_{i=1}^{n} S_{i}$ follow IG distribution with parameters $\left(n m \mu, n^{2} m \lambda\right)$, where the subscript ' $k$ ' denotes the $k^{\text {th }}(1,2,3)$ case of ASP and each $S_{i}(i=1,2,3, \ldots, n)$ are 
'm' times of its initial stress $\left(S_{i}=m Y_{i}\right)$ with distribution as $I G(m \mu, m \lambda)$. The probability density function of augmented strength $\left(Z_{k}\right)$ under generalized case of ASP can be given as

$$
f_{z_{k}}(z)=\sqrt{\frac{n^{2} m \lambda}{2 \pi z_{k}{ }^{3}}} \exp -\left\{\frac{n^{2} m \lambda\left(z_{k}-n m \mu\right)^{2}}{2 n^{2} m^{2} \mu^{2} z_{k}}\right\}, z_{k} \geq 0, \mu>0, \lambda>0, m>0, n>1
$$

where ' $m$ ' is positive real number and ' $n$ ' is positive integer. A general form of augmented strength reliability $\left(R_{k}\right)$ under the generalized case of ASP is obtained as

$$
R_{k}=P\left(Z_{k}>Y\right)=\frac{n}{2} \sqrt{\frac{m \lambda}{2 \pi}} e^{\frac{n \lambda}{\mu}}\left[\begin{array}{l}
\int_{0}^{\infty} \operatorname{erfc}\left(\frac{\sqrt{\frac{\lambda}{z_{k}}}\left(-z_{k}+\mu\right)}{\sqrt{2} \mu}\right) z^{-3 / 2} \exp \left\{\frac{-\lambda z_{k}}{2 m \mu^{2}}-\frac{n^{2} m \lambda}{2 z_{k}}\right\} d z_{k} \\
+e^{2 \lambda / \mu} \int_{0}^{\infty} \operatorname{erfc}\left(\frac{\sqrt{\frac{\lambda}{z_{k}}}\left(z_{k}+\mu\right)}{\sqrt{2} \mu}\right) z^{-3 / 2} \exp \left\{\frac{-\lambda z_{k}}{2 m \mu^{2}}-\frac{n^{2} m \lambda}{2 z_{k}}\right\} d z_{k}
\end{array}\right]
$$

where $\operatorname{erfc}($.$) is complementary function of error function \operatorname{erf}($.$) , which can be$ defined as

$$
\begin{aligned}
& \operatorname{erfc}(x)=1-\operatorname{erf}(x) \text { and } \\
& \operatorname{erf}(x)=\frac{2}{\sqrt{\pi}} \int_{0}^{x} e^{-t^{2}} d t .
\end{aligned}
$$

\section{Maximum Likelihood Estimation of Generalized Augmented Strength Reliability:}

This section deals with the maximum likelihood estimation of parameters of $R_{k}$. Suppose that $\mathbf{Z}_{\mathbf{k}}=\left\{Z_{k 1}, Z_{k 2}, \ldots, Z_{k n_{1}}\right\}$ and $\mathbf{Y}=\left\{Y_{1}, Y_{2}, \ldots, Y_{n_{2}}\right\}$ be the two independent random samples of sizes $n_{1}$ and $n_{2}$ are drawn from the augmented inverse Gaussian strength and inverse Gaussian stress distributions respectively. Then the generalized form of likelihood and log-likelihood functions based on the observed random samples are respectively defined as

$$
\begin{aligned}
L_{k}\left(\mu, \lambda / z_{k}, y\right) & =n^{n_{1}} m^{\frac{n_{1}}{2}}\left(\frac{\lambda}{2 \pi}\right)^{\frac{n_{1}+n_{2}}{2}} \prod_{i=1}^{n_{1}} z_{k i}(-3 / 2) \prod_{j=1}^{n_{2}} y_{j}^{(-3 / 2)} \\
& \exp \left[-\frac{\lambda}{2 \mu^{2}}\left(\left\{\frac{n_{1} \bar{z}_{k}}{m}+n_{2} \bar{y}\right)-2 \mu\left(n n_{1}+n_{2}\right)+\mu^{2}\left(n^{2} m \sum_{i=1}^{n_{1}}\left(\frac{1}{z_{k i}}\right)+\sum_{j=1}^{n_{2}}\left(\frac{1}{y_{j}}\right)\right)\right\}\right]
\end{aligned}
$$




$$
\begin{array}{r}
\log L_{k}\left(\mu, \lambda / z_{k}, y\right)=n_{1} \log n+\frac{n_{1}}{2} \log m+\frac{\left(n_{1}+n_{2}\right)}{2}(\log \lambda-\log 2 \pi)-\frac{3}{2} \sum_{i=1}^{n_{1}} \log z_{k i}-\frac{3}{2} \sum_{j=1}^{n_{2}} \log y_{j} \\
-\frac{\lambda}{2 \mu^{2}}\left\{\left(\frac{n_{1} \bar{z}_{k}}{m}+n_{2} \bar{y}\right)-2 \mu\left(n n_{1}+n_{2}\right)+\mu^{2}\left(n^{2} m \sum_{i=1}^{n_{1}}\left(\frac{1}{z_{k i}}\right)+\sum_{j=1}^{n_{2}}\left(\frac{1}{y_{j}}\right)\right)\right\}
\end{array}
$$

The likelihood equations with respect to $\mu$ and $\lambda$ are given by

$$
\frac{\partial \log L_{k}\left(\mu, \lambda / z_{k}, y\right)}{\partial \mu}=0 \text { and } \frac{\partial \log L_{k}\left(\mu, \lambda / z_{k}, y\right)}{\partial \lambda}=0
$$

The maximum likelihood estimators $\hat{\mu}$ and $\hat{\lambda}$ of $\mu$ and $\lambda$ are respectively given as

$$
\begin{aligned}
& \hat{\mu}=\frac{\frac{n_{1} \overline{z_{k}}}{m}+n_{2} \bar{y}}{n n_{1}+n_{2}} \text { and } \\
& \hat{\lambda}=\frac{n_{1}+n_{2}}{\left(n^{2} m \sum_{i=1}^{n_{1}}\left(\frac{1}{z_{k i}}\right)+\sum_{j=1}^{n_{2}}\left(\frac{1}{y_{j}}\right)\right)-\frac{\left(n n_{1}+n_{2}\right)^{2}}{\left(\frac{n_{1} \overline{z_{k}}}{m}+n_{2} \bar{y}\right)}}
\end{aligned}
$$

Thus the maximum likelihood estimate of generalized form of augmented strength reliability $\left(\hat{R}_{k}\right)$ can be obtained through its invariance property by substituting $\hat{\mu}$ and $\hat{\lambda}$ in place of $\mu$ and $\lambda$ in the expression of $R_{k}$.

Remark: The MLE of augmented strength reliability $\left(R_{k}\right)$ for Cases-I, II and III under ASP can be obtained directly by substituting $k=n=1 ; k=2, m=1$; and $k=3$ respectively in the expression of $\hat{R}_{k}$.

\section{Bayesian Estimation of Generalized Augmented Strength Reliability Models:}

In this section, we propose Bayes estimation of $R_{k}(k=1,2,3)$ for the generalized case of ASP by assuming the model parameters $\mu$ and $\lambda$ as independent random variables with non-informative types of priors. In Bayesian paradigm, [5] argued that if there are no information or less information is available regarding to the model parameters then it is better to consider a non-informative prior for the unknown model parameters than informative priors. In this study we consider non-informative (uniform and Jeffrey's) types of priors by assuming that no information is known priori about the underlying parameters of interest. For a comprehensive comparison of the proposed Bayes estimators, we consider two different loss functions known as symmetric (SELF) and asymmetric (LLF). In fact, the squared error loss function considers the overestimation and underestimation as equally penalized, whereas, in LINEX loss function the overestimation is more serious than the underestimation or vice-versa. 


\subsection{Choosing Uniform prior:}

In this study, considering $\mu$ and $\lambda$ are independent random variables having noninformative uniform type of priors. The joint prior probability density function of $\mu$ and $\lambda$ is given by

$$
g_{1}(\mu, \lambda)=\frac{1}{\mu \lambda} \quad ; \quad 0<\mu, \lambda<\infty
$$

The joint posterior density function can be found by taking convolution of the likelihood function $L_{k}\left(\mu, \lambda / z_{k}, y\right)$ and the joint prior probability density function $g_{1}(\mu, \lambda)$. The joint posterior probability distribution of $\mu$ and $\lambda$ is given as

$\prod_{k 1}(\mu, \lambda /$ data $)=K g_{1}(\mu, \lambda) L_{k}(\mu, \lambda /$ data $)$.

Where $K$ is normalizing constant which is defined as

$$
K^{-1}=\int_{0}^{\infty} \int_{0}^{\infty} g_{1}(\mu, \lambda) L_{k}(\mu, \lambda / \text { data }) d \mu d \lambda
$$

Here, we consider two different loss functions, viz. Squared Error Loss Function (SELF) and Linex Loss Function (LLF) for better comprehension of Bayesian analysis. The Bayes estimator of any parametric function, say $\varphi(\mu, \lambda)$ under SELF as well as LLF are respectively defined by

$$
\begin{aligned}
\hat{\varphi}(\mu, \lambda)^{\text {self }} & =E[\varphi(\mu, \lambda) / \text { data }]=\int_{(\mu, \lambda)} \varphi(\mu, \lambda) \Pi_{k 1}(\mu, \lambda / \text { data }) d \mu d \lambda \\
\hat{\varphi}(\mu, \lambda)^{l l f} & =-\frac{1}{p} \log \{E[\exp \{-p \cdot \varphi(\mu, \lambda)\} / \text { data }]\} \\
& =\frac{-1}{p} \log \left[\int_{(\mu, \lambda)} \exp \{-p \cdot \varphi(\mu, \lambda)\} \Pi_{k 1}(\mu, \lambda / \text { data }) d \mu d \lambda\right]
\end{aligned}
$$

The joint posterior probability distribution under uniform prior is given as

$$
\prod_{k 1}(\mu, \lambda / \text { data }) \propto \mu^{-1} \lambda^{\frac{n_{1}+n_{2}}{2}-1} \exp \left[-\frac{\lambda}{2 \mu^{2}}\left\{\left(\frac{n_{1} \bar{z}_{k}}{m}+n_{2} \bar{y}\right)-2 \mu\left(n n_{1}+n_{2}\right)+\mu^{2}\left(n^{2} m \sum_{i=1}^{n_{1}}\left(\frac{1}{z_{k i}}\right)+\sum_{j=1}^{n_{2}}\left(\frac{1}{y_{j}}\right)\right)\right\}\right]
$$

The Bayes estimator of generalized augmented strength reliability under squared error loss function is given as

$$
\begin{aligned}
& \hat{R}_{k}^{\text {self }}=\int_{(\alpha, \lambda)} R_{k} \Pi_{k 1}(\mu, \lambda / \text { data }) d \mu d \lambda \\
& \propto \int_{0}^{\infty} \int_{0}^{\infty} R_{k} \mu^{-1} \lambda^{\frac{n_{1}+n_{2}}{2}-1} \exp \left[-\frac{\lambda}{2 \mu^{2}}\left\{\left(\frac{n_{1} \bar{z}_{k}}{m}+n_{2} \bar{y}\right)-2 \mu\left(n n_{1}+n_{2}\right)+\mu^{2}\left(n^{2} m \sum_{i=1}^{n_{1}}\left(\frac{1}{z_{k i}}\right)+\sum_{j=1}^{n_{2}}\left(\frac{1}{y_{j}}\right)\right)\right\}\right] \partial \mu \partial \lambda
\end{aligned}
$$


Where $R_{k}$ is the augmented strength reliability model for generalized case of ASP, given in equation (3).

Under Linex loss function the Bayes estimate of augmented strength reliability $R_{k}(k=1,2,3)$ for generalized case of ASP is given as

$$
\begin{aligned}
& \hat{R}_{k}^{\text {Ilf }}=\frac{-1}{p} \ln \left[E\left(e^{-p R_{k}} / \text { data }\right)\right]=\frac{-1}{p} \ln \left[\iint_{0}^{\infty} e_{0}^{-p R_{k}} \Pi_{k 1}(\mu, \lambda / \text { data }) d \mu d \lambda\right] \\
& \propto \frac{-1}{p} \ln \left[\int_{0}^{\infty} \int_{0}^{\infty} e^{-p R_{k}} \mu^{-1} \lambda^{\frac{n_{1}+n_{2}}{2}-1} \exp \left[-\frac{\lambda}{2 \mu^{2}}\left\{\left(\frac{n_{1} \bar{z}_{k}}{m}+n_{2} \bar{y}\right)-2 \mu\left(n n_{1}+n_{2}\right)+\mu^{2}\left(n^{2} m \sum_{i=1}^{n_{1}}\left(\frac{1}{z_{k i}}\right)+\sum_{j=1}^{n_{2}}\left(\frac{1}{y_{j}}\right)\right)\right\}\right] \partial \mu \partial \lambda\right]
\end{aligned}
$$

The expressions obtained above is not in explicit form and cannot be evaluated manually, thus the numerical approximations through Markov-Chain Monte-Carlo (MCMC) have been done to evaluate the above expression.

\subsection{Choosing Jeffrey's prior:}

Considering the parameters $\mu$ and $\lambda$ as independent random variables having noninformative Jeffrey's prior. [18] proposed a type of non-informative prior, which is defined as

$$
J(\alpha, \lambda) \propto \sqrt{\operatorname{det} I(\alpha, \lambda)}
$$

where, $I(\alpha, \lambda)$ is Fisher information matrix, defined as follow

$I(\alpha, \lambda)=-\left[\begin{array}{cc}E\left(\frac{\partial^{2} \log L}{\partial \alpha^{2}}\right) & E\left(\frac{\partial^{2} \log L}{\partial \alpha \partial \lambda}\right) \\ E\left(\frac{\partial^{2} \log L}{\partial \lambda \partial \alpha}\right) & E\left(\frac{\partial^{2} \log L}{\partial \lambda^{2}}\right)\end{array}\right]=\left[\begin{array}{cc}\frac{\lambda\left(n n_{1}+n_{2}\right)}{\mu^{3}} & 0 \\ 0 & \frac{\left(n_{1}+n_{2}\right)}{2 \lambda^{2}}\end{array}\right]$

Thus, the joint Jeffrey prior of $\mu$ and $\lambda$ is given as

$$
\begin{aligned}
J(\alpha, \lambda) & =\left[\frac{\left(n_{1}+n_{2}\right)\left(n n_{1}+n_{2}\right)}{2}\right]^{1 / 2}\left(\mu^{3} \lambda\right)^{-1 / 2} \\
& \propto\left(\mu^{3} \lambda\right)^{-1 / 2}
\end{aligned}
$$

The joint posterior distribution of $\alpha$ and $\lambda$ is defined by

$$
\prod_{k 2}(\mu, \lambda / \text { data }) \propto \mu^{-3 / 2} \lambda^{\frac{n_{1}+n_{2}-1}{2}} \exp \left[-\frac{\lambda}{2 \mu^{2}}\left\{\left(\frac{n_{1} \bar{z}_{k}}{m}+n_{2} \bar{y}\right)-2 \mu\left(n n_{1}+n_{2}\right)+\mu^{2}\left(n^{2} m \sum_{i=1}^{n_{1}}\left(\frac{1}{z_{k i}}\right)+\sum_{j=1}^{n_{2}}\left(\frac{1}{y_{j}}\right)\right)\right\}\right]
$$

Thus, the Bayes estimator of augmented strength reliability under squared error loss function is defined as the posterior means of $R_{k}(k=1,2,3)$, given by 
$\hat{R}_{k}^{\text {self }} \propto \int_{0}^{\infty} \int_{0}^{\infty} R_{k} \mu^{-3 / 2} \lambda^{\frac{n_{1}+n_{2}-1}{2}} \exp \left[-\frac{\lambda}{2 \mu^{2}}\left\{\left(\frac{n_{1} \bar{z}_{k}}{m}+n_{2} \bar{y}\right)-2 \mu\left(n n_{1}+n_{2}\right)+\mu^{2}\left(n^{2} m \sum_{i=1}^{n_{1}}\left(\frac{1}{z_{k i}}\right)+\sum_{j=1}^{n_{2}}\left(\frac{1}{y_{j}}\right)\right)\right\}\right] d \alpha d \lambda$

The Bayes estimate $\left(\hat{R}_{k}{ }^{\text {llf }}\right)$ of augmented strength reliability $\operatorname{model}\left(R_{k} ; k=1,2,3\right)$ for Jeffrey's prior under linex loss function, given by

$\hat{R}_{k}{ }^{\text {If }} \propto \frac{-1}{p} \ln \left[\int_{0}^{\infty} \int_{0}^{\infty} e^{-p R_{k}} \mu^{-3 / 2} \lambda^{\frac{n_{1}+n_{2}-1}{2}} \exp \left[-\frac{\lambda}{2 \mu^{2}}\left\{\left(\frac{n_{1} \bar{z}_{k}}{m}+n_{2} \bar{y}\right)-2 \mu\left(n n_{1}+n_{2}\right)+\mu^{2}\left(n^{2} m \sum_{i=1}^{n_{1}}\left(\frac{1}{z_{k i}}\right)+\sum_{j=1}^{n_{2}}\left(\frac{1}{y_{j}}\right)\right)\right\}\right] d \alpha d \lambda\right]$

It is noticed that all the expressions of Bayes estimators augmented strength reliability $\left(R_{k} ; k=1,2,3\right)$ obtained in equations in (15) and (16) under uniform prior as well as in (21) and (22) under Jeffrey's prior are not in explicit form and cannot be evaluated analytically. Hence as an alternatively numerical method viz., Markov Chain Monte Carlo (MCMC) sampling procedure (see; $[6,17]$ ) is used to approximate the integrals. We therefore, used Metropolis-Hastings algorithm to sample from the posterior distribution $\prod_{k}(\mu, \lambda /$ data $)$.

\section{Data Analysis:}

In this section we performed the data analysis for simulated as well as real data sets to demonstrate the applicability of the above proposed estimators (ML and Bayes) of augmented strength reliability under generalized case of ASP.

Example 1: In this example we consider the simulated data analysis of augmented strength reliability. We generate two separate samples of sizes 30 each from strength and stress distribution $I G\left(n m \mu, n^{2} m \lambda\right)$ and $I G(\mu, \lambda)$ respectively with parameters $\mu=3.5, \lambda=0.5, m=2$ and $\mathrm{n}=2$. The true value for augmented strength reliability for case-III is given as $R_{3}=0.81894$. The maximum likelihood estimate of augmented strength reliability $\left(R_{3}\right)$ is given by $\hat{R}_{3}=0.7915405$. The Bayes estimate of augmented strength reliability under squared error and linex loss functions separately for both uniform and Jeffrey's priors are computed through Metropolis-Hastings numerical approximation algorithm with ten thousand random generations of $\mu$ and $\lambda$ by considering normal density as candidate density. Thus the Bayes estimate of augmented strength reliability for uniform prior under SELF (LLF) is 0.7983433 (0.7979621) . Similarly, the Bayes estimate of augmented strength reliability for Jeffrey's prior under SELF (LLF) is 0.7984137(0.7980516) .

Example 2: this example deals with the fitting and analyzing the real life data set initially reported by [16]. The considered data sets represent the survival times of two groups of patients suffering from the head and neck cancer complications. The patients in one group were diagnosed using Radiotherapy (RT), whereas the other group patients were diagnosed using Radiotherapy and Chemotherapy (RT+CT). Recently, [20] have attempted to analyze head-neck cancer (HNC) data for log-Normal distribution in Bayesian paradigm and reported that the model is useful for such type of problems. Latter, 
[25] considered same data sets and analyzed for Inverse Lindley distribution (ILD) and compared the fitting results with Inverse Rayleigh distribution (IRD). The data sets are presented below as:

Table 1. Survival times of patients diagnosed using RT: Data X.

\begin{tabular}{cccccccccc}
\hline 6.53 & 7 & 10.42 & 14.48 & 16.1 & 22.7 & 34 & 41.55 & 42 & 45.28 \\
49.4 & 53.62 & 63 & 64 & 83 & 84 & 91 & 108 & 112 & 129 \\
133 & 133 & 139 & 140 & 140 & 146 & 149 & 154 & 157 & 160 \\
160 & 165 & 146 & 149 & 154 & 157 & 160 & 160 & 165 & 173 \\
176 & 218 & 225 & 241 & 248 & 273 & 277 & 297 & 405 & 417 \\
420 & 440 & 523 & 583 & 594 & 1101 & 1146 & 1417 & & \\
\hline
\end{tabular}

\begin{tabular}{cccccccccc}
\multicolumn{10}{c}{ Table 2. Survival times of patients diagnosed using CT+RT: Data Y. } \\
\hline 12.2 & 23.56 & 23.74 & 25.87 & 31.98 & 37 & 41.35 & 47.38 & 55.46 & 58.36 \\
63.47 & 68.46 & 78.26 & 74.47 & 81.43 & 84 & 92 & 94 & 110 & 112 \\
119 & 127 & 130 & 133 & 140 & 146 & 155 & 159 & 173 & 179 \\
194 & 195 & 209 & 249 & 281 & 319 & 339 & 432 & 469 & 519 \\
633 & 725 & 817 & 1776 & & & & & & \\
\hline
\end{tabular}

Here we wanted to make use of this data under the assumption of two parameters IG distribution. We, therefore, tested the goodness of fit of the data sets for two parameter IG distribution using Kolmogorov-Smirnov (KS) statistic, Akaike information criterion (AIC) and Bayesian information criterion (BIC). We compare the applicability of the data sets for IG distribution with ILD, which have similar characteristics of upside-down bathtub shaped hazard curve. The fitting summary is presented in Table 3 and the empirical and fitted CDF's for both the data sets are given in Figure 1, which clearly shows that the IG distribution better fits to the concern data sets than ILD. Thus, using above data sets the maximum likelihood estimates of augmented strength reliability $\left(R_{3}\right)$ is given by $\hat{R}_{3}=0.7884156$.

Table 3: Data Fitting summary

\begin{tabular}{|c|c|c|c|c|c|}
\hline Data & Model & K-S & $\begin{array}{c}\text { Log } \\
\text {-Likelihood }\end{array}$ & AIC & BIC \\
\hline Data1 & \multirow{2}{*}{ IGD } & 0.15985 & -386.2751 & 776.5503 & 780.6712 \\
Data2 & 0.084951 & -278.5416 & 561.0832 & 564.6516 \\
\hline Data1 & ILD & 0.76792 & -476.8458 & 955.6916 & 957.7521 \\
Data2 & 0.78904 & -359.3569 & 720.7137 & 722.4979 \\
\hline
\end{tabular}



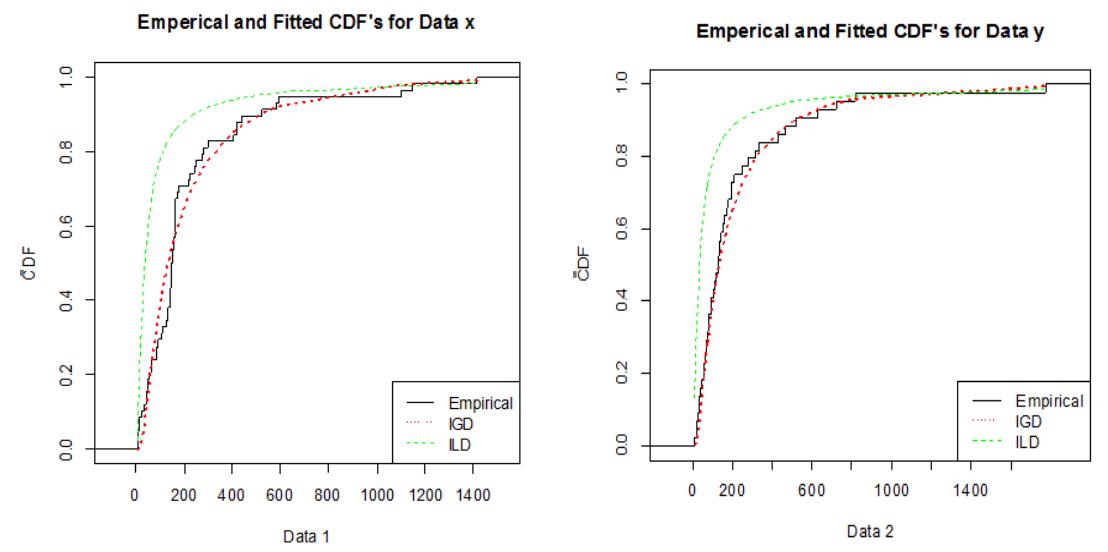

Figure 1. Empirical and fitted CDF's for IGD and ILD for Data X and Data Y

The Bayes estimates of augmented strength reliability under squared error and Linex loss functions for uniform and Jeffrey's priors separately have been carried out through Metropolis-Hasting algorithm. Ten thousands of random samples of $\mu$ and $\lambda$ have drawn from the posterior density by considering Normal density as candidate density. The Bayes estimates under different loss functions have been calculated using the ten thousand random samples of $\mu$ and $\lambda$. Thus the Bayes estimate of augmented strength reliability for uniform prior under SELF (LLF) is 0.7929581 (0.7925881). Similarly, the Bayes estimate of augmented strength reliability for Jeffrey's prior under SELF (LLF) is $0.7938642(0.7935042)$.

\section{Simulation Study and Discussions:}

This section presents the validation and comparison of the different methods of estimators (ML and Bayes) through simulation study for different combinations of sample sizes and stress-strength reliability parameters with 1000 replications. The Bayes estimators of generalized augmented strength reliability for two different loss functions (i.e. SELF and LLF) under uniform and Jeffery's priors have been compared with that of ML estimators through their mean square errors and absolute biases. It may be noticed that the expressions of posterior expectations for both the priors are not in closed form, thus to find out numerical evaluation, MCMC technique viz. Metropolis-Hastings algorithm has been used for drawing the sample from the arbitrary posterior distribution. For generating a random sample of size $N$ (say) from a posterior distribution $\pi(\boldsymbol{\theta} /$ data $) ; \boldsymbol{\theta}=(\mu, \lambda)^{\prime}$, the basic Metropolis-Hastings algorithm consist the following steps:

1. Choose initial value for the parameter $\boldsymbol{\theta}^{(0)}=\left(\mu^{(0)}, \lambda^{(0)}\right)$ such that $\pi\left(\boldsymbol{\theta}^{(0)}\right)>0$.

2. For $j=1,2, \ldots, N$ repeat the following steps

i. Set $\boldsymbol{\theta}=\boldsymbol{\theta}^{(j-1)}$

ii. Draw a 'candidate' value $\boldsymbol{\theta}^{c}$ from a proposal density say $q\left(\boldsymbol{\theta}^{c} / \boldsymbol{\theta}\right)$.

iii. Generate ' $U$ ' uniform variate on range 0 and 1 i.e., $u \sim U(0,1)$. 
iv. If $u \leq \min (1, R)$, accept the candidate point $\left(\boldsymbol{\theta}^{c}\right)$ with probability

$$
\min \left\{1, R=\frac{\pi\left(\boldsymbol{\theta}^{c} / \text { data }\right) q\left(\boldsymbol{\theta} / \boldsymbol{\theta}^{c}\right)}{\pi(\boldsymbol{\theta} / \text { data }) q\left(\boldsymbol{\theta}^{c} / \boldsymbol{\theta}\right)}\right\} \text {, otherwise set } \boldsymbol{\theta}^{(j)}=\boldsymbol{\theta} \text {. }
$$

Here, $q$ is the transition probability matrix of the Markov chain with same support as that of likelihood function and $q\left(\boldsymbol{\theta}^{c} / \boldsymbol{\theta}\right)$ is the transition probability from $\boldsymbol{\theta}$ to $\boldsymbol{\theta}^{\mathbf{c}}$.

To evaluate the Bayes estimate of augmented strength reliability model ten thousand random samples have been drawn by following the above steps of Metropolis-Hastings algorithm by considering asymptotic normal distribution as proposal distribution. The initial values of the chain are fixed as the ML estimates of the parameters along with their asymptotic variance covariance matrix to draw the initial random samples from proposal density. The first thousand samples have been discarded as burn-in period of Markov chain. The autocorrelation of the chain has also been tested and it is found that the chains are unfortunately highly auto correlated. To reduce the present autocorrelation within the chain, we trimmed every second simulation.

The following given tables 4-7 contain the average estimates, MSE and absolute biases for MLE and Bayes estimates for uniform as well as Jeffrey's prior under each of SELF and LLF of generalized augmented strength reliability under ASP for different values of stress-strength reliability parameters $\mu, \lambda, n$ and $m$ and the sample sizes $\left(n_{1}, n_{2}\right)$. The following observations are made based on the given tables.

Table 4: avg, MSE and abs. Bias for estimates of $R_{k}$ for variation of $\mu$

\begin{tabular}{|c|c|c|c|c|c|c|}
\hline \multicolumn{7}{|c|}{$\mu=2.5, \quad \mathrm{R}=0.928036$} \\
\hline \multirow{2}{*}{$\left(n_{1}, n_{2}\right)$} & \multirow[t]{2}{*}{ Statistic } & \multirow[t]{2}{*}{ MLE } & \multicolumn{2}{|c|}{ Uniform prior } & \multicolumn{2}{|c|}{ Jeffrey's prior } \\
\hline & & & SELF & LLF & SELF & LLF \\
\hline \multirow{3}{*}{$(10,10)$} & \multirow{3}{*}{$\begin{array}{c}\text { avg. } \\
\text { MSE } \\
\text { abs. bias }\end{array}$} & 0.930544 & 0.925624 & 0.925012 & 0.928882 & 0.928335 \\
\hline & & 0.000556 & 0.000481 & 0.000492 & 0.000462 & 0.000469 \\
\hline & & 0.002508 & 0.002413 & 0.003024 & 0.000845 & 0.000299 \\
\hline \multirow{3}{*}{$(20,30)$} & \multirow{3}{*}{$\begin{array}{c}\text { avg. } \\
\text { MSE } \\
\text { abs. bias }\end{array}$} & 0.926726 & 0.924688 & 0.924431 & 0.926083 & 0.925838 \\
\hline & & 0.00024 & 0.000227 & 0.000229 & 0.000221 & 0.000223 \\
\hline & & 0.00099 & 0.001048 & 0.001305 & 0.000347 & 0.000102 \\
\hline \multirow{3}{*}{$(30,20)$} & \multirow{3}{*}{$\begin{array}{c}\text { avg. } \\
\text { MSE } \\
\text { abs. bias }\end{array}$} & 0.918885 & 0.917027 & 0.916764 & 0.918346 & 0.918096 \\
\hline & & 0.000253 & 0.000238 & 0.00024 & 0.000234 & 0.000236 \\
\hline & & 0.000621 & 0.001237 & 0.001499 & $8.28 \mathrm{E}-05$ & 0.000168 \\
\hline \multirow{3}{*}{$(50,50)$} & \multirow{3}{*}{$\begin{array}{c}\text { avg. } \\
\text { MSE } \\
\text { abs. bias }\end{array}$} & 0.924347 & 0.923326 & 0.923206 & 0.923973 & 0.923856 \\
\hline & & 0.000116 & 0.000111 & 0.000112 & 0.000111 & 0.000112 \\
\hline & & 0.000611 & 0.00041 & 0.00053 & 0.000236 & 0.000119 \\
\hline \multicolumn{7}{|c|}{$\mu=4.5, \quad R=0.879886$} \\
\hline \multirow{2}{*}{$\left(n_{1}, n_{2}\right)$} & \multirow[t]{2}{*}{ Statistic } & \multirow[t]{2}{*}{ MLE } & \multicolumn{2}{|c|}{ Uniform prior } & \multicolumn{2}{|c|}{ Jeffrey's prior } \\
\hline & & & SELF & LLF & SELF & LLF \\
\hline
\end{tabular}




\begin{tabular}{|c|c|c|c|c|c|c|}
\hline$(10,10)$ & $\begin{array}{c}\text { avg. } \\
\text { MSE } \\
\text { abs. bias }\end{array}$ & $\begin{array}{l}0.883677 \\
0.001251 \\
0.003791\end{array}$ & $\begin{array}{l}0.885055 \\
0.001013 \\
0.005169\end{array}$ & $\begin{array}{c}0.884198 \\
0.00102 \\
0.004312\end{array}$ & $\begin{array}{l}0.888608 \\
0.001061 \\
0.008722\end{array}$ & $\begin{array}{l}0.887832 \\
0.001062 \\
0.007946\end{array}$ \\
\hline$(20,30)$ & $\begin{array}{c}\text { avg. } \\
\text { MSE } \\
\text { abs. bias }\end{array}$ & $\begin{array}{l}0.884641 \\
0.000451 \\
0.001416\end{array}$ & $\begin{array}{c}0.88523 \\
0.000409 \\
0.002005\end{array}$ & $\begin{array}{l}0.884864 \\
0.000411 \\
0.001638\end{array}$ & $\begin{array}{l}0.886807 \\
0.000414 \\
0.003581\end{array}$ & $\begin{array}{l}0.886458 \\
0.000414 \\
0.003233\end{array}$ \\
\hline$(30,20)$ & $\begin{array}{c}\text { avg. } \\
\text { MSE } \\
\text { abs. bias }\end{array}$ & $\begin{array}{l}0.889951 \\
0.000417 \\
0.000575\end{array}$ & $\begin{array}{l}0.890368 \\
0.000376 \\
0.000992\end{array}$ & $\begin{array}{l}0.890049 \\
0.000378 \\
0.000673\end{array}$ & $\begin{array}{l}0.891892 \\
0.000381 \\
0.002515\end{array}$ & $\begin{array}{l}0.891586 \\
0.000382 \\
0.002209\end{array}$ \\
\hline$(50,50)$ & $\begin{array}{c}\text { avg. } \\
\text { MSE } \\
\text { abs. bias }\end{array}$ & $\begin{array}{l}0.888143 \\
0.000224 \\
0.000252\end{array}$ & $\begin{array}{l}0.888436 \\
0.000214 \\
4.07 \mathrm{E}-05\end{array}$ & $\begin{array}{l}0.888276 \\
0.000214 \\
0.000119\end{array}$ & $\begin{array}{l}0.889144 \\
0.000213 \\
0.000749\end{array}$ & $\begin{array}{l}0.888987 \\
0.000214 \\
0.000591\end{array}$ \\
\hline \multicolumn{7}{|c|}{$\mu=6.5, \quad R=0.853085$} \\
\hline$\left(n_{1}, n_{2}\right)$ & Statistic & MLE & $\begin{array}{c}\text { Unifor } \\
\text { SELF }\end{array}$ & prior & $\begin{array}{l}\text { Jeffrey } \\
\text { SELF }\end{array}$ & $\frac{\text { s prior }}{\text { LLF }}$ \\
\hline$(10,10)$ & $\begin{array}{c}\text { avg. } \\
\text { MSE } \\
\text { abs. bias }\end{array}$ & $\begin{array}{l}0.857459 \\
0.001436 \\
0.004374\end{array}$ & $\begin{array}{c}0.86339 \\
0.001216 \\
0.010304\end{array}$ & $\begin{array}{l}0.862456 \\
0.001213 \\
0.009371\end{array}$ & $\begin{array}{l}0.866634 \\
0.001308 \\
0.013549\end{array}$ & $\begin{array}{c}0.865786 \\
0.0013 \\
0.0127\end{array}$ \\
\hline$(20,30)$ & $\begin{array}{c}\text { avg. } \\
\text { MSE } \\
\text { abs. bias }\end{array}$ & $\begin{array}{l}0.859319 \\
0.000576 \\
0.001373\end{array}$ & $\begin{array}{c}0.861987 \\
0.00053 \\
0.004042\end{array}$ & $\begin{array}{c}0.861579 \\
0.00053 \\
0.003634\end{array}$ & $\begin{array}{l}0.863629 \\
0.000542 \\
0.005683\end{array}$ & $\begin{array}{l}0.863235 \\
0.000541 \\
0.005289\end{array}$ \\
\hline$(30,20)$ & $\begin{array}{c}\text { avg. } \\
\text { MSE } \\
\text { abs. bias }\end{array}$ & $\begin{array}{c}0.864194 \\
0.00051 \\
0.001607\end{array}$ & $\begin{array}{c}0.866527 \\
0.000473 \\
0.00394\end{array}$ & $\begin{array}{c}0.866167 \\
0.000473 \\
0.00358\end{array}$ & $\begin{array}{l}0.867982 \\
0.000482 \\
0.005395\end{array}$ & $\begin{array}{c}0.867637 \\
0.00048 \\
0.00505\end{array}$ \\
\hline$(50,50)$ & $\begin{array}{c}\text { avg. } \\
\text { MSE } \\
\text { abs. bias }\end{array}$ & $\begin{array}{l}0.875051 \\
0.000276 \\
0.001409\end{array}$ & $\begin{array}{c}0.876002 \\
0.000262 \\
0.00236\end{array}$ & $\begin{array}{l}0.875821 \\
0.000262 \\
0.002179\end{array}$ & $\begin{array}{l}0.876883 \\
0.000268 \\
0.003242\end{array}$ & $\begin{array}{l}0.876707 \\
0.000268 \\
0.003065\end{array}$ \\
\hline
\end{tabular}

Table 5: avg, MSE and abs. Bias for estimates of $R_{k}$ for variation of $\lambda$

When $\mu=3.5 ; m=n=2$

\begin{tabular}{|c|c|c|c|c|c|c|}
\hline \multicolumn{7}{|c|}{$\lambda=\mathbf{1 . 5}, \mathbf{R}=\mathbf{0 . 8 6 7 0 7 3}$} \\
\hline \multirow{2}{*}{$\left(n_{1}, n_{2}\right)$} & Statistic & MLE & \multicolumn{2}{c|}{ Uniform prior } & \multicolumn{2}{c|}{ Jeffrey's prior } \\
\cline { 4 - 7 } & & & SELF & LLF & SELF & LLF \\
\hline \multirow{3}{*}{$(10,10))$} & avg. & 0.870502 & 0.873945 & 0.873034 & 0.877771 & 0.876948 \\
& MSE & 0.001276 & 0.001068 & 0.001071 & 0.001115 & 0.001111 \\
& abs. bias & 0.003429 & 0.006872 & 0.005961 & 0.010698 & 0.009875 \\
\hline \multirow{3}{*}{$(20,30)$} & avg. & 0.869891 & 0.871714 & 0.871319 & 0.87342 & 0.873042 \\
& MSE & 0.000603 & 0.000553 & 0.000553 & 0.000569 & 0.000568 \\
& abs. bias & 0.002281 & 0.004104 & 0.003709 & 0.00581 & 0.005432 \\
\hline
\end{tabular}




\begin{tabular}{|c|c|c|c|c|c|c|}
\hline$(30,20)$ & $\begin{array}{c}\text { avg. } \\
\text { MSE } \\
\text { abs. bias }\end{array}$ & $\begin{array}{l}0.880035 \\
0.000468 \\
0.000854\end{array}$ & $\begin{array}{c}0.881705 \\
0.00042 \\
0.002524\end{array}$ & $\begin{array}{l}0.881379 \\
0.000421 \\
0.002198\end{array}$ & $\begin{array}{c}0.883081 \\
0.000431 \\
0.0039\end{array}$ & $\begin{array}{l}0.882768 \\
0.000431 \\
0.003587\end{array}$ \\
\hline$(50,50)$ & $\begin{array}{c}\text { avg. } \\
\text { MSE } \\
\text { abs. bias }\end{array}$ & $\begin{array}{l}0.876012 \\
0.000245 \\
0.001144\end{array}$ & $\begin{array}{l}0.876794 \\
0.000233 \\
0.001926\end{array}$ & $\begin{array}{l}0.876603 \\
0.000233 \\
0.001736\end{array}$ & $\begin{array}{l}0.877588 \\
0.000236 \\
0.002721\end{array}$ & $\begin{array}{l}0.877401 \\
0.000235 \\
0.002534\end{array}$ \\
\hline \multicolumn{7}{|c|}{$\lambda=4.5, \quad R=0.945831$} \\
\hline$\left(n_{1}, n_{2}\right)$ & Statistic & MLE & $\begin{array}{c}\text { Unifo } \\
\text { SELF }\end{array}$ & $\frac{\text { p prior }}{\text { LLF }}$ & $\begin{array}{l}\text { Jeffrey } \\
\text { SELF }\end{array}$ & $\frac{\text { s prior }}{\text { LLF }}$ \\
\hline$(10,10)$ & $\begin{array}{c}\text { avg. } \\
\text { MSE } \\
\text { abs. bias }\end{array}$ & $\begin{array}{c}0.94859 \\
0.00049 \\
0.002759\end{array}$ & $\begin{array}{l}0.941959 \\
0.000466 \\
0.003873\end{array}$ & $\begin{array}{l}0.941456 \\
0.000479 \\
0.004375\end{array}$ & $\begin{array}{c}0.94503 \\
0.000428 \\
0.000801\end{array}$ & $\begin{array}{l}0.944586 \\
0.000438 \\
0.001245\end{array}$ \\
\hline$(20,30)$ & $\begin{array}{c}\text { avg. } \\
\text { MSE } \\
\text { abs. bias }\end{array}$ & $\begin{array}{l}0.945416 \\
0.000231 \\
0.000333\end{array}$ & $\begin{array}{l}0.942571 \\
0.000232 \\
0.002511\end{array}$ & $\begin{array}{l}0.942371 \\
0.000235 \\
0.002712\end{array}$ & $\begin{array}{l}0.943909 \\
0.000219 \\
0.001174\end{array}$ & $\begin{array}{l}0.943721 \\
0.000221 \\
0.001362\end{array}$ \\
\hline$(30,20)$ & $\begin{array}{c}\text { avg. } \\
\text { MSE } \\
\text { abs. bias }\end{array}$ & $\begin{array}{l}0.940101 \\
0.000208 \\
0.000283\end{array}$ & $\begin{array}{c}0.937569 \\
0.000209 \\
0.00225\end{array}$ & $\begin{array}{l}0.937385 \\
0.000212 \\
0.002433\end{array}$ & $\begin{array}{c}0.938747 \\
0.0002 \\
0.001071\end{array}$ & $\begin{array}{l}0.938573 \\
0.000202 \\
0.001246\end{array}$ \\
\hline$(50,50)$ & $\begin{array}{c}\text { avg. } \\
\text { MSE } \\
\text { abs. bias }\end{array}$ & $\begin{array}{l}0.943228 \\
0.000108 \\
0.000423\end{array}$ & $\begin{array}{l}0.941841 \\
0.000108 \\
0.000964\end{array}$ & $\begin{array}{l}0.941748 \\
0.000109 \\
0.001057\end{array}$ & $\begin{array}{l}0.942432 \\
0.000106 \\
0.000372\end{array}$ & $\begin{array}{l}0.942341 \\
0.000106 \\
0.000463\end{array}$ \\
\hline
\end{tabular}

Table 6: avg, MSE and abs. Bias for estimates of $R_{k}$ for variation of $n$ When $\mu=3.5 ; \lambda=2.5 ; m=2$

\begin{tabular}{|c|c|c|c|c|c|c|}
\hline \multicolumn{7}{|c|}{$\mathbf{n}=\mathbf{2}, \quad \mathbf{R}=\mathbf{0 . 9 0 0 9 2 9}$} \\
\hline \multirow{2}{*}{$\left(n_{1}, n_{2}\right)$} & \multirow[t]{2}{*}{ Statistic } & \multirow[t]{2}{*}{ MLE } & \multicolumn{2}{|c|}{ Uniform prior } & \multicolumn{2}{|c|}{ Jeffrey's prior } \\
\hline & & & SELF & LLF & $\overline{\text { SELF }}$ & LLF \\
\hline \multirow{3}{*}{$(10,10)$} & $\begin{array}{l}\text { avg. } \\
\text { MSE }\end{array}$ & 0.904094 & 0.902674 & 0.901903 & 0.906064 & 0.905373 \\
\hline & IVIDL & 0.001034 & 0.000829 & 0.000841 & 0.00084 & 0.000846 \\
\hline & & 0.003165 & 0.001745 & 0.000974 & 0.005135 & 0.004444 \\
\hline \multirow{3}{*}{$(20,30)$} & avg. & 0.901485 & 0.900893 & 0.900572 & 0.902474 & 0.902168 \\
\hline & MSE & 0.000438 & 0.000398 & 0.000400 & 0.000401 & 0.000403 \\
\hline & & 0.001385 & 0.000793 & 0.000471 & 0.002374 & 0.002067 \\
\hline \multirow{3}{*}{$(30,20)$} & avg. & 0.897095 & 0.896735 & 0.896442 & 0.898072 & 0.897789 \\
\hline & IVIDL & 0.000375 & 0.000341 & 0.000342 & 0.00035 & 0.00035 \\
\hline & & 0.002142 & 0.001782 & 0.001489 & 0.003119 & 0.002836 \\
\hline \multirow{3}{*}{$(50,50)$} & avg. & 0.895924 & 0.895766 & 0.895609 & 0.896477 & 0.896323 \\
\hline & MSE & 0.000201 & 0.000193 & 0.000194 & 0.000192 & 0.000192 \\
\hline & & 0.000885 & 0.000726 & 0.000569 & 0.001437 & 0.001283 \\
\hline \multicolumn{7}{|c|}{$\mathrm{n}=4, \quad \mathrm{R}=0.984549$} \\
\hline \multirow{2}{*}{$\left(n_{1}, n_{2}\right)$} & \multirow[t]{2}{*}{ Statistic } & \multirow[t]{2}{*}{ MLE } & \multicolumn{2}{|c|}{ Uniform prior } & \multicolumn{2}{|c|}{ Jeffrey's prior } \\
\hline & & & SELF & LLF & SELF & LLF \\
\hline
\end{tabular}




\begin{tabular}{|c|c|c|c|c|c|c|}
\hline$(10,10)$ & $\begin{array}{c}\text { avg. } \\
\text { MSE } \\
\text { abs. bias }\end{array}$ & $\begin{array}{l}0.984742 \\
9.05 \mathrm{E}-05 \\
0.000193\end{array}$ & $\begin{array}{l}0.980038 \\
0.000121 \\
0.004511\end{array}$ & $\begin{array}{l}0.979915 \\
0.000123 \\
0.004634\end{array}$ & $\begin{array}{l}0.981808 \\
9.70 \mathrm{E}-05 \\
0.002741\end{array}$ & $\begin{array}{l}0.981707 \\
9.87 \mathrm{E}-05 \\
0.002842\end{array}$ \\
\hline$(20,30)$ & $\begin{array}{c}\text { avg. } \\
\text { MSE } \\
\text { abs. bias }\end{array}$ & $\begin{array}{l}0.982436 \\
4.49 \mathrm{E}-05 \\
5.17 \mathrm{E}-05\end{array}$ & $\begin{array}{l}0.980478 \\
5.07 \mathrm{E}-05 \\
0.001907\end{array}$ & $\begin{array}{l}0.980432 \\
5.12 \mathrm{E}-05 \\
0.001953\end{array}$ & $\begin{array}{l}0.981188 \\
4.60 \mathrm{E}-05 \\
0.001196\end{array}$ & $\begin{array}{l}0.981145 \\
4.64 \mathrm{E}-05 \\
0.001239\end{array}$ \\
\hline$(30,20)$ & $\begin{array}{c}\text { avg. } \\
\text { MSE } \\
\text { abs. bias }\end{array}$ & $\begin{array}{l}0.984288 \\
3.06 \mathrm{E}-05 \\
7.51 \mathrm{E}-06\end{array}$ & $\begin{array}{l}0.982608 \\
3.52 \mathrm{E}-05 \\
0.001688\end{array}$ & $\begin{array}{l}0.982578 \\
3.54 \mathrm{E}-05 \\
0.001718\end{array}$ & $\begin{array}{c}0.983135 \\
3.24 \mathrm{E}-05 \\
0.00116\end{array}$ & $\begin{array}{l}0.983107 \\
3.26 \mathrm{E}-05 \\
0.001189\end{array}$ \\
\hline$(50,50)$ & $\begin{array}{c}\text { avg. } \\
\text { MSE } \\
\text { abs. bias }\end{array}$ & $\begin{array}{c}0.98315 \\
0.00002 \\
0.0002\end{array}$ & $\begin{array}{l}0.98223 \\
0.00002 \\
0.00071\end{array}$ & $\begin{array}{l}0.98221 \\
0.00002 \\
0.00073\end{array}$ & $\begin{array}{l}0.98248 \\
0.00002 \\
0.00046\end{array}$ & $\begin{array}{l}0.98247 \\
0.00002 \\
0.00048\end{array}$ \\
\hline \multicolumn{7}{|c|}{$\mathrm{n}=8, \quad \mathrm{R}=0.99912$} \\
\hline$\left(n_{1}, n_{2}\right)$ & Statistic & MLE & $\begin{array}{l}\text { Unifor } \\
\text { SELF }\end{array}$ & $\frac{1 \text { prior }}{\text { LLF }}$ & $\begin{array}{l}\text { Jeffrey } \\
\text { SELF }\end{array}$ & $\frac{\text { s prior }}{\text { LLF }}$ \\
\hline$(10,10)$ & $\begin{array}{c}\text { avg. } \\
\text { MSE } \\
\text { abs. bias }\end{array}$ & $\begin{array}{l}0.998956 \\
1.53 \mathrm{E}-06 \\
0.000164\end{array}$ & $\begin{array}{l}0.997624 \\
6.03 \mathrm{E}-06 \\
0.001496\end{array}$ & $\begin{array}{l}0.997617 \\
6.09 \mathrm{E}-06 \\
0.001503\end{array}$ & $\begin{array}{l}0.997978 \\
4.39 \mathrm{E}-06 \\
0.001142\end{array}$ & $\begin{array}{l}0.997972 \\
4.42 \mathrm{E}-06 \\
0.001148\end{array}$ \\
\hline$(20,30)$ & $\begin{array}{c}\text { avg. } \\
\text { MSE } \\
\text { abs. bias }\end{array}$ & $\begin{array}{l}0.999288 \\
3.46 \mathrm{E}-07 \\
6.19 \mathrm{E}-05\end{array}$ & $\begin{array}{l}0.998905 \\
8.01 \mathrm{E}-07 \\
0.000445\end{array}$ & $\begin{array}{l}0.998904 \\
8.03 \mathrm{E}-07 \\
0.000446\end{array}$ & $\begin{array}{l}0.998987 \\
6.64 \mathrm{E}-07 \\
0.000363\end{array}$ & $\begin{array}{l}0.998986 \\
6.66 \mathrm{E}-07 \\
0.000364\end{array}$ \\
\hline$(30,20)$ & $\begin{array}{c}\text { avg. } \\
\text { MSE } \\
\text { abs. bias }\end{array}$ & $\begin{array}{l}0.999175 \\
3.01 \mathrm{E}-07 \\
7.83 \mathrm{E}-06\end{array}$ & $\begin{array}{l}0.998791 \\
6.60 \mathrm{E}-07 \\
0.000392\end{array}$ & $\begin{array}{c}0.99879 \\
6.62 \mathrm{E}-07 \\
0.000392\end{array}$ & $\begin{array}{l}0.998873 \\
5.45 \mathrm{E}-07 \\
0.000309\end{array}$ & $\begin{array}{c}0.998873 \\
5.46 \mathrm{E}-07 \\
0.00031\end{array}$ \\
\hline$(50,50)$ & $\begin{array}{c}\text { avg. } \\
\text { MSE } \\
\text { abs. bias }\end{array}$ & $\begin{array}{l}0.999002 \\
2.72 \mathrm{E}-07 \\
7.32 \mathrm{E}-05\end{array}$ & $\begin{array}{c}0.99879 \\
4.21 \mathrm{E}-07 \\
0.000286\end{array}$ & $\begin{array}{l}0.998789 \\
4.21 \mathrm{E}-07 \\
0.000286\end{array}$ & $\begin{array}{c}0.99883 \\
3.84 \mathrm{E}-07 \\
0.000245\end{array}$ & $\begin{array}{c}0.99883 \\
3.85 \mathrm{E}-07 \\
0.000246\end{array}$ \\
\hline
\end{tabular}

Table 7: avg, MSE and abs. Bias for estimates of $R_{k}$ for variation of $\mathrm{m}$ When $\mu=3.5 ; \lambda=2.5 ; n=2$

\begin{tabular}{|c|c|c|c|c|c|c|}
\hline \multicolumn{7}{|c|}{$\mathrm{m}=5, \mathrm{R}=\mathbf{0 . 9 7 8 8 8 9}$} \\
\hline \multirow[t]{2}{*}{$\left(n_{1}, n_{2}\right)$} & \multirow[t]{2}{*}{ Statistic } & \multirow[t]{2}{*}{ MLE } & \multicolumn{2}{|c|}{ Uniform prior } & \multicolumn{2}{|c|}{ Jeffrey's prior } \\
\hline & & & SELF & LLF & SELF & LLF \\
\hline \multirow{3}{*}{$(10,10)$} & \multirow{3}{*}{$\begin{array}{c}\text { avg. } \\
\text { MSE } \\
\text { abs. bias }\end{array}$} & 0.97886 & 0.974028 & 0.973816 & 0.976454 & 0.97629 \\
\hline & & 0.000141 & 0.000172 & 0.000177 & 0.000135 & 0.000138 \\
\hline & & $2.89 \mathrm{E}-05$ & 0.004861 & 0.005072 & 0.002434 & 0.002598 \\
\hline \multirow{3}{*}{$(20,30)$} & \multirow{3}{*}{$\begin{array}{c}\text { avg. } \\
\text { MSE } \\
\text { abs. bias }\end{array}$} & 0.981238 & 0.979409 & 0.979352 & 0.980194 & 0.980142 \\
\hline & & $4.98 \mathrm{E}-05$ & $5.54 \mathrm{E}-05$ & $5.60 \mathrm{E}-05$ & $5.02 \mathrm{E}-05$ & $5.07 \mathrm{E}-05$ \\
\hline & & 4.01E-06 & 0.001825 & 0.001881 & 0.00104 & 0.001092 \\
\hline \multirow{3}{*}{$(30,20)$} & \multirow{3}{*}{$\begin{array}{c}\text { avg. } \\
\text { MSE } \\
\text { abs. bias }\end{array}$} & 0.979799 & 0.978078 & 0.978026 & 0.978803 & 0.978755 \\
\hline & & 5.03E-05 & 5.34E-05 & $5.39 \mathrm{E}-05$ & 4.95E-05 & 4.99E-05 \\
\hline & & 0.000308 & 0.001412 & 0.001465 & 0.000688 & 0.000736 \\
\hline
\end{tabular}




\begin{tabular}{|c|c|c|c|c|c|c|}
\hline$(50,50)$ & $\begin{array}{c}\text { avg. } \\
\text { MSE } \\
\text { abs. bias }\end{array}$ & $\begin{array}{l}0.980535 \\
2.53 \mathrm{E}-05 \\
2.70 \mathrm{E}-05\end{array}$ & $\begin{array}{l}0.979511 \\
2.70 \mathrm{E}-05 \\
0.000997\end{array}$ & $\begin{array}{l}0.979484 \\
2.71 \mathrm{E}-05 \\
0.001023\end{array}$ & $\begin{array}{c}0.979938 \\
2.54 \mathrm{E}-05 \\
0.00057\end{array}$ & $\begin{array}{l}0.979913 \\
2.55 \mathrm{E}-05 \\
0.000594\end{array}$ \\
\hline \multicolumn{7}{|c|}{$\mathrm{m}=7, \mathbf{R}=\mathbf{0 . 9 9 1 8 7 3}$} \\
\hline \multirow{2}{*}{$\left(n_{1}, n_{2}\right)$} & \multirow[t]{2}{*}{ Statistic } & \multirow[t]{2}{*}{ MLE } & \multicolumn{2}{|c|}{ Uniform prior } & \multicolumn{2}{|c|}{ Jeffrey's prior } \\
\hline & & & SELF & LLF & SELF & LLF \\
\hline \multirow{3}{*}{$(10,10)$} & \multirow{3}{*}{$\begin{array}{c}\text { avg. } \\
\text { MSE } \\
\text { abs. bias }\end{array}$} & 0.991332 & 0.987457 & 0.987356 & 0.989066 & 0.988993 \\
\hline & & $4.57 \mathrm{E}-05$ & $8.18 \mathrm{E}-05$ & $8.42 \mathrm{E}-05$ & $5.77 \mathrm{E}-05$ & $5.91 \mathrm{E}-05$ \\
\hline & & 0.000541 & 0.004416 & 0.004517 & 0.002807 & 0.00288 \\
\hline \multirow{3}{*}{$(20,30)$} & \multirow{3}{*}{$\begin{array}{c}\text { avg. } \\
\text { MSE } \\
\text { abs. bias }\end{array}$} & 0.991949 & 0.990473 & 0.99045 & 0.99102 & 0.991 \\
\hline & & $1.91 \mathrm{E}-05$ & $2.53 \mathrm{E}-05$ & $2.56 \mathrm{E}-05$ & $2.13 \mathrm{E}-05$ & $2.15 \mathrm{E}-05$ \\
\hline & & 0.000228 & 0.001704 & 0.001728 & 0.001158 & 0.001178 \\
\hline \multirow{3}{*}{$(30,20)$} & \multirow{3}{*}{$\begin{array}{c}\text { avg. } \\
\text { MSE } \\
\text { abs. bias }\end{array}$} & 0.991442 & 0.990073 & 0.990051 & 0.990568 & 0.99055 \\
\hline & & $2.00 \mathrm{E}-05$ & $2.43 \mathrm{E}-05$ & $2.45 \mathrm{E}-05$ & $2.14 \mathrm{E}-05$ & $2.16 \mathrm{E}-05$ \\
\hline & & $6.71 \mathrm{E}-05$ & 0.001303 & 0.001324 & 0.000807 & 0.000825 \\
\hline \multirow{3}{*}{$(50,50)$} & \multirow{3}{*}{$\begin{array}{c}\text { avg. } \\
\text { MSE } \\
\text { abs. bias }\end{array}$} & 0.991696 & 0.99099 & 0.990981 & 0.991229 & 0.991221 \\
\hline & & $8.73 \mathrm{E}-06$ & $9.76 \mathrm{E}-06$ & $9.80 \mathrm{E}-06$ & $9.20 \mathrm{E}-06$ & $9.23 \mathrm{E}-06$ \\
\hline & & 0.000102 & 0.000604 & 0.000613 & 0.000365 & 0.000373 \\
\hline
\end{tabular}

* In Table 4, the effects of variation of $\mu(2.5,4.5,6.5)$ have been presented for the fixed value of other model parameters $\lambda=2.5 ; n=m=2$. It is observed from the table that the Bayes estimate of augmented strength reliability for generalized case of ASP gives better result than that of MLE on the basis of MSE and absolute biases. In comparison between the Bayes estimate for both the priors, it is noticed that the Bayes estimate for uniform prior under squared error loss function have the minimum mean square error and absolute biases. Moreover it is also seen that the true value of augmented strength reliability under generalized case of ASP decreases for higher values of $\mu$. It is observed that the mean square errors and absolute biases gradually decrease for the increasing values of sample sizes $\left(n_{1}, n_{2}\right)$.

* The variation of $\lambda(1.5,4.5)$ have been reported by fixing rest model parameters $\mu=3.5 ; n=m=2$ in Table 5. It may be noticed from the table that the true augmented strength reliability increases with increasing value of $\lambda$. It is observed that the Bayes estimates for uniform and Jeffery's prior under both the loss functions are producing very close estimates to the augmented strength reliability. The pattern of MSE and absolute biases are decreasing in nature for increasing values of sample sizes. Overall, Bayes estimates, specifically under uniform prior, gives more precise results in comparison with MLE. 
* In Table 6 , the variation of $n(2,4,8)$ have been shown by fixing other model parameters $\mu=3.5 ; \lambda=2.5 ; m=2$ and it is observed that the true generalized augmented strength reliability increases with increasing values of $n$ (number of added components), i.e. the strength reliability is enhanced by adding more components. It may also be observed from the table that the Bayes estimators give better result than MLE for small value of $n(2)$ and vice-versa for higher values of $n(4,8)$.

* Table 7 presents the variation of $m(5,7)$ by keeping other parameters fixed as $\mu=3.5 ; \lambda=2.5 ; n=2$ and it is observed that the strength reliability get enhanced up to $99 \%$ for higher values of $m$. Here it may be noticed that the Bayes estimators for Jeffery's prior gives closer results to ML estimators as compared with that of uniform prior. It is also observe that the Bayes estimate for Jeffery's prior under SELF gives good estimates for small value of $m(5)$ but for higher values of $m(7)$, the MLE performs better. Overall, it is also seen that the mean square errors and absolute biases gradually decrease for increasing values of sample sizes.

\section{Conclusions:}

In this article, Bayes and ML estimation of augmented strength reliability models under ASP for inverse Gaussian distribution is considered. The Bayes estimation is particularly focused on non-informative (uniform and Jeffrey's) types of priors under the SELF and LLF. A numerical comparison between ML and Bayes estimators are carried out on the basis of their mean square errors and absolute biases. It is noticed from the simulation analysis that the proposed Bayes estimates of augmented strength reliability perform efficiently in compare to ML estimates. Finally, it may be suggestive that the Bayes estimators can perform better if the choice of priori information is more adequate.

Further, there are some open problems for future research on Bayes estimation for complete and censored samples of augmenting strength reliability when the stress and strength are independent but not identically distributed.

\section{Acknowledgments:}

The authors would like to thanks the editor and referees for giving their fruitful comments to improve the standard of article and also thankful to the University Grants Commission, Govt. of India, New Delhi, India for providing financial support to carry out the proposed work under the major research project (ref no. F. 42-38/2013 (SR) dated March 12 $2^{\text {th }}$, 2013).

\section{References}

[1]. Alam, S.N. and Roohi. On Augmenting Exponential Strength-Reliability. IAPQR Transactions, 2002; 27: 111-117. 
[2]. Banerjee, A. K. and G. K. Bhattacharyya. Bayesian results for the inverse Gaussian distribution with an application. Technometrics, 1979; 21(2): 247-251.

[3]. Basu, A. P. and N Ebrahimi. On the reliability of stochastic systems. Statistics \& Probability Letters, 1983; 1(5): 265-267.

[4]. Basu, S. and R. T. Lingham. Bayesian estimation of system reliability in Brownian stressstrength models. Annals of the Institute of Statistical Mathematics, 2003; 55(1): 7-19.

[5]. Berger, J.O. Statistical Decision Theory and Bayesian Analysis. Springer-Verlag: NY; 1985.

[6]. Brooks, S. Markov chain Monte Carlo method and its application. Journal of the royal statistical society: series D (the Statistician). 1998; 47(1): 69-100.

[7]. Chandra, N. and S. Sen. Augmented Strength Reliability of Equipment under Gamma Distribution. J of Statist. Theory and Applications. 2014; 13: 212-221.

[8]. Chandra, N. and V.K Rathaur. Augmented Strategy Plans for Enhancing Strength Reliability of an Equipment under Inverse Gaussian Distribution. J. Math. Engg. Sci. Aerospace: Special Issue on Reliability and Dependability Modeling Analysis for Complex Systems. 2015(a); 6: 233-243.

[9]. Chandra, N. and V.K Rathaur. Augmenting Exponential Stress-Strength Reliability for a coherent system. Proceedings of National Seminar on Statistical Methods and Data Analysis, published by Abhiruchi Prakashana, Mysore. 2015(b):25-34.

[10]. Chandra, N. and V.K Rathaur. Augmented Gamma Strength Reliability Models for Series and Parallel Coherent System. Proceedings of National Conference on Emerging Trends in Statistical Research: Issue and Challenges, Narosa Publication, New Delhi, India, 2015(c):43-54.

[11]. Chhikara, R.S. and J.L. Folks. Estimation of the Inverse Gaussian distribution function. J. Amer. Statis. Assoc. 1974; 69: 250-254.

[12]. Chhikara, R.S. and J.L. Folks. Statistical distributions related to the inverse Gaussian. Communications in Statistics. 1975; 4: 1081-1091.

[13]. Chhikara, R.S. and J.L. Optimum test procedures for the mean of first passage time distributions in Brownian motion with positive drift. Technometrics. 1976; 18:189-193.

[14]. Chhikara, R.S. and J.L. The Inverse Gaussian distribution as a Lifetime Model. Technometrics. $1977 ;$ 19(4): 461-468.

[15]. Ebrahimi, N. and T. Ramallingam. Estimation of system reliability in Brownian stressstrength models based on sample paths. Annals of the Institute of Statistical Mathematics. 1993; 45(1): 9-19.

[16]. Efron, B. Logistic regression, survival analysis and the Kaplan-meier curve. J. Ame. Stat. Asso. 1988; 83: 414-425.

[17]. Hastings, W.K. Monte Carlo sampling methods using Markov chains and their applications. Biometrika. 1970; 57(1): 97-109.

[18]. Jeffreys, H. The Theory of Probability. $3^{\text {rd }}$ ed, Oxford University Press, New York, NY.(1998).

[19]. Johnson, N. L., S. Kotz and N Balakrishnan. Continuous univariate distributions. (2 ${ }^{\text {nd }}$ ed.), vol.1 John Wiley \& Sons. New York, 163: 1994.

[20]. Makkar, P., P.K. Srivastava, R.S. Singh and S.K. Upadhyay. Bayesian survival analysis of head and neck cancer data using lognormal model. Communications in Statistics-Theory and Methods. 2014; 43: 392-407.

[21]. Nadas, A. Best tests for zero drift based on first passage times in Brownian motion. Technometrics. 1973; 15: 125-132.

[22]. Padgett, W. J. and L.J. Wei. Estimation for the three-parameter inverse Gaussian distribution. Communications in statistics-theory and methods. 1979; 8(2): 129-137.

[23]. Pandey, B.N. and P. Bandyopadhyay. Bayesian Estimation of Inverse Gaussian Distribution. Int. J. Agricult. Stat. Sci. 2013; 9(2): 373-386.

[24]. Sarhan, A.M., B. Smith and D.C. Hamilton. Estimation of $P(Y<X)$ for a Two-parameter Bathtub Shaped Failure Rate Distribution. Int. J. of Statist. Prob. 2015; 4: 33-45. 
[25]. Sharma,V.K., S.K. Singh, U. Singh and V. Agiwal. The inverse Lindley distribution: a stress-strength reliability model with application to head and neck cancer data. J. of Indust. and Product. Engg. 2015; 32: 162-173.

[26]. Sherif, Y.S. and M. L. Smith. First-passage time distribution of Brownian motion as a reliability model. IEEE Transactions. 1980; 29(5): 425-426.

[27]. Tweedie, M. C. K. Statistical Properties of Inverse Gaussian distribution I, Annals of Mathematics \& Statistics. 1957(a); 28: 362-77.

[28]. Tweedie, M. C. K. Statistical properties of inverse Gaussian distributions II. Annals of Mathematical Statistics. 1957(b); 28(3): 696-705.

Dr. Navin Chandra is an Assistant Professor of Statistics in the Department of Statistics, Ramanujan School of Mathematical Sciences at Pondicherry University, Pondicherry, India. He has been awarded for Master of Science (Statistics) and Doctor of Philosophy in Statistics discipline from Banaras Hindu University, Varanasi, India. Dr. Chandra is recipient of Senior Research Fellowship in a major research program sponsored by Department of Science and Technology, Government of India, New Delhi, India. He is presently working on a major research project sponsored by University Grants Commission, New Delhi, India. His research interests includes in Bayesian Inference, Reliability Theory- Accelerated Life Testing, Stress-Strength Models, Augmentation Strength Reliability Models, and in Survival Analysis.

Mr. Vikash Kumar Rathaur has received M.Sc. (Statistics) in 2013 and then subsequently he is currently pursuing for Ph.D. degree in Statistics at Department of Statistics, Ramanujan School of Mathematical Sciences at Pondicherry University, Pondicherry, India. He received fellowship in a major research project sponsored by University Grants Commission, New Delhi, India. His research interest is in Bayesian and Reliability Theory. 